\title{
Operativo Ginebra. La dirigencia sindical ante la instalación internacional de la dictadura militar (1976)
}

\author{
Luciana Zorzoli \\ University of London - UNLP - Conicet - zorzoli@gmail.com
}

El golpe de Estado producido por las Fuerzas Armadas en marzo de 1976 inauguró una serie de cambios radicales. La transformación económica acompañó el comienzo del giro neoliberal mientras que el establecimiento de una politica sistemática de violación de los derechos humanos, desaparición de opositores y robo de bebés transformó una sociedad que se habia caracterizado por su movilización política y cultural.

En el marco de esa reestructuración el gobierno militar consideró prioritaria la transformación del movimiento obrero buscando eliminar los sectores clasistas que revelaron su extensión a partir de las jornadas de mayo de 1969 en la provincia de Córdoba (Brennan y Gordillo, 2008; Pozzi y Schneider, 2000). La represión de los trabajadores, especialmente delegados de base y activistas, se hizo en forma invariable a nivel nacional acompañada de un fuerte disciplinamiento que restringia la capacidad de organización y de respuesta. El tratamiento que recibieron los sindicatos estuvo marcado por ese contexto represivo y por la reincidente búsqueda de cercenar su poder por parte de los militares. A la intervención de la Confederación General del Trabajo (CGT) y de un grupo de importantes sindicatos peronistas se sumó el impedimento de ejercer derechos consolidados como el de huelga y el de negociación colectiva, mientras se decidia la suspensión de gran parte de la legislación laboral existente (Zorzoli, 2015).

Este artículo sin embargo considera que en los trabajos clásicos sobre sindicatos y dictadura primó, y aún se encuentra vigente, una identificación entre lo sucedido en el movimiento sindical de base y lo sucedido en la dirigencia sindical que requiere ser revisada. ${ }^{1}$ Por eso se

1. Una versión preliminar de este trabajo fue debatida en las Jornadas "Regular y legislar el mundo obrero latinoamericano. Aportes para una historia regional de la OIT" (2015) en el IdIHCS (UNLP - Conicet). Agradezco aquí los comentarios de V. Basualdo y A. Stagnaro y la lectura posterior de A. Schneider.

(Archivos, año IV, nº 8, marzo de 2016, pp. 13-32) 
propone, en el contexto de aportar a la construcción de una explicación alternativa sobre "que pasó con los sindicatos" durante el período, revisar el posicionamiento de la dirigencia sindical cuando debe producirse la participación en la Organización Internacional del Trabajo (OIT).

En esta revisión se refutarán los análisis en los que se ha construido la imagen de un sindicalismo cuya actividad estuvo "suspendida" por la represión (en especial Delich, 1982; Abós, 1984) y se indagará en la importancia, muchas veces mencionada, de las participaciones en la OIT. ${ }^{2}$

Realizar esta reconstrucción para el año 1976 permitirá examinar el momento de "instalación" del plan represivo y colaborar en el análisis de las razones de su éxito. Permitirá además no adjudicar posicionamientos ex post a la dirigencia sindical, como se ha hecho asumiendo que la dinámica principal fue "quietud" entre 1976 y 1979, y "conflicto" entre 1979 y $1983 .^{3}$

Como introducción se considerará el interés dictatorial en la participación en la OIT. En la segunda parte del artículo se realiza una crónica y caracterización de los sectores sindicales que viajaron a Ginebra y de sus posicionamientos tanto a nivel local como en el exterior, con una mención de lo que acontece entre los grupos no confederados. En el último apartado se presentan las conclusiones preliminares de este estudio.

\section{La Argentina en el exterior en tiempos de cambios radicales}

Desde el 24 de marzo de 1976 hasta los últimos actos de su gestión la dictadura que derrocó al gobierno de Isabel Perón se ocupó de su imagen externa prestando especial atención a la repercusión que pudieran tener dos temas: la cuestión de los derechos humanos y la situación laboral. Su objetivo era evitar que las denuncias sobre el régimen represivo y la suspensión sine die de muchos de los derechos sindicales tuvieran un impacto negativo sobre el gobierno, algo que pesaba ya para otras dictaduras latinoamericanas, especialmente la de Augusto Pinochet. Entre 1981 y 1982 se sumaría a esta agenda la defensa de los derechos territoriales argentinos cuando en el país comenzaba la

2. Aunque las menciones al tema son constantes en los estudios del periodo, hasta ahora no hubo un abordaje específico del tema y se toma como referencia lo indicado por Abós, que señala que la OIT fue un "espacio de denuncia" (Abós, 1984: 42-47, 95-109).

3. Uno de los pocos trabajos que escapa a este esquema es el que presentó A. Fernández (1985) que contenía una elaboración matizada de la situación vivida entre 1976 y 1983 y partía de una diferenciación analítica clave de la que carecian muchos de los trabajos previos: la situación de los sectores obreros, incluso de los movilizados, no era equiparable a la de la dirigencia sindical. 
disputa por establecer "la legitimidad de lo actuado" por la Junta a lo largo de todo el proceso.

Puede conjeturarse que esa preocupación por la imagen de Argentina estaba constituida por razones de economía y política internacional pero también por el impacto que esos cuestionamientos pudieran tener localmente. El conocimiento interno de esos cuestionamientos, que el gobierno militar se ocupó de desacreditar etiquetándolos como una "campaña antiargentina", 4 podia funcionar activando o legitimando denuncias que fueron constitutivas de las respuestas sociales al terrorismo de Estado, como la llevada adelante por los familiares de personas desaparecidas. Esa preocupación hizo que mientras se reprimía localmente el gobierno presentara en el exterior lo que hacía y sucedía en la Argentina como un problema interno. Éste se resolvería, insistian, para dar paso a una "democracia moderna a tono con las naciones avanzadas del mundo occidental”.

Para la presentación de este diagnóstico y la legitimación de esos objetivos dos espacios fueron centrales, las Naciones Unidas y su Comisión de Derechos Humanos y la OIT y su Comité de Libertad Sindical, donde actúo diligentemente el embajador Gabriel Martínez. ${ }^{5}$ En este último organismo centraremos nuestra atención, pues este espacio funcionó como articulador de la relación sindicatos-gobierno y en él se hizo evidente el posicionamiento de la dirigencia sindical, objeto de este trabajo.

\section{La instalación internacional del gobierno militar y la posición sindical}

Un día después de que las Fuerzas Armadas tomaran el control operativo del país, el 25 de marzo de 1976, se presentó ante la OIT la

4. La denuncia de que existía tal campaña comienza en el contexto del primer viaje a la OIT. La realiza entre otros el ministro del Interior, Gral. Harguindeguy, señalando en los medios que "existe una campaña muy bien dirigida desde el exterior para desprestigiar a las actuales autoridades" (ver La Nación y Clarín, 3 de junio de 1976).

5. Se trata del economista Gabriel Martínez, embajador extraordinario y plenipotenciario en la Misión Permanente de la República Argentina ante los Organismos Internacionales en Ginebra, que fuera nombrado en 1974 por Perón (Decretos $1.080 / 74$ y $1.081 / 74$ ) y que continuara sus funciones con una destacada actividad a favor del "Proceso" hasta noviembre de 1983 (Novaro y Palermo, 2003: 282-283). Martínez había hecho una rápida carrera diplomática, fue asesor económico de la embajada en Chile, tuvo posiciones diplomáticas en Bonn y Bruselas, fue jefe del Departamento de Comercio y secretario de Comercio Internacional, desde donde se señala tuvo una relación con López Rega (Guest, 1990: 103-110). Su participación en el tercer gobierno peronista no lo invalidó para continuar con sus tareas; por el contrario, la Junta Militar encontró en él a uno de sus más destacados estrategas. 
primera queja formal por la situación represiva que se desplegaba contra las organizaciones obreras argentinas. ${ }^{6}$

La misma fue tramitada, siguiendo los procedimientos de la OIT, como una queja ante el Comité de Libertad Sindical presentada por la Federación Sindical Mundial (FSM). En ella se denunciaba a las autoridades militares por haber intervenido la CGT y suspendido las actividades sindicales. Según consta en el telegrama -que es el primer documento del caso abierto contra Argentina bajo el número $842-,{ }^{7}$ la FSM añadía que la sede del Unión Obrera Metalúrgica (UOM) en Buenos Aires había sido tomada y que un número no especificado de dirigentes habian sido detenidos, incluido su secretario general, Lorenzo Miguel. A esta primera denuncia se sumó una de la Confederación Internacional de Sindicatos Libres (CISL) junto con otras entre las que estaban las de la Unión Internacional de Sindicatos de Trabajadores Metalúrgicos, de la Federación Internacional Sindical de la Enseñanza y una copia de una nota enviada a Videla por la Organización Regional Interamericana de Trabajadores el 25 de marzo en la que pedian al gobierno que respetara los convenios internacionales en la materia.

En una segunda comunicación en abril, la FSM incorporaba mayores detalles de la situación argentina, denunciaba que se habia suspendido el derecho de huelga y que continuaban las intervenciones. Indicaba que las detenciones se habian ampliado e incorporaba un listado provisorio de ocho dirigentes en esa situación. Informaba asimismo de situaciones que iban más allá de Buenos Aires, como la detención en Córdoba de trabajadores metalúrgicos o las condenas dictadas por los consejos de guerra en las provincias.

A esta primera queja -que iría ampliándose- se añadía la realizada el 9 de abril por la Confederación Mundial del Trabajo (CMT) que pedía la intervención de la OIT y reclamaba la liberación de los sindicalistas y el restablecimiento de la libertad de asociación. Igualmente la CMT sumaba una lista de dirigentes detenidos y de sindicatos que se encontraban bajo intervención y denunciaba que a este escenario habia que sumar una serie de represalias patronales. En junio añadia denuncias específicas (a la Compañia Ploghi de Córdoba o la General Motors de

6. Sobre los antecedentes de las relaciones internacionales del movimiento sindical argentino pueden verse entre otros Belloni Ravest (1969), Godio (1980), Godio y Wachendorfer (1986), Parcero (1987), Alexander (2009) y Basualdo (2010).

7. De aquí en más todas las menciones sobre el caso sin indicación en contrario se basan en el trabajo realizado en el Archivo de la OIT en Ginebra, Suiza. Para su mejor identificación se utiliza la siguiente signatura propuesta por esta investigación: Archivo Organización Internacional del Trabajo, Caso 842, Comité de Libertad Sindical, $\mathrm{n}^{\circ}$ de carpeta/año. Se propone como forma abreviada: A-OIT, C842, $\mathrm{n}^{\circ}$ de carpeta/año. En el caso de esta cita corresponde: A-OIT, Caso 842, 1/1976. 
Barracas) y protestaba contra la política de fijación de salarios difundida por el ministro Martínez de Hoz.

Siguiendo los mecanismos de la OIT, cada una de estas quejas fue informada al gobierno argentino, que envió el 20 de mayo su primer respuesta al organismo. En ella Martinez informaba a la OIT que las Fuerzas Armadas habían asumido la conducción del Estado para garantizar el cumplimiento de la justicia y el pleno respeto de los derechos humanos (un argumento que invertía la denuncia señalando a la "subversión" como responsable de la violación de los mismos). Anunciaba que se estaba llevando a cabo una reorganización nacional y que en ese contexto "se ha dispuesto que en adelante las organizaciones sindicales ajusten el ejercicio de sus funciones a la defensa de las legitimas aspiraciones de sus integrantes, evitando incursionar en áreas ajenas a su competencia", señalando que "como primera etapa en el proceso de saneamiento de la actividad gremial -afectada también por el desorden, la corrupción y la subversión- y a los fines de reencauzarla en las vías del orden y la justicia, y siempre al servicio del interés nacional, fue suspendida transitoriamente, con excepción de la correspondiente a la administración interna de las entidades sindicales y a la de sus obras sociales" (ver A-OIT, Caso 842, 1/1976, Carta de la Misión Permanente de la Rep. Argentina ante los Organismos Internacionales en Ginebra).

A esa respuesta el gobierno militar adjuntaba la nueva Ley de Contratos de Trabajo y el discurso de Horacio Liendo del 1 de mayo e indicaba que había intervenido temporariamente 28 sindicatos sobre un total de 1.368 , lo que era presentado como argumento a favor de la excepcionalidad de las medidas tomadas, pues representarian solo el 2,04\% del total. Si bien la cifra de sindicatos indicada (1.368) es muy superior a otras más fiables que indican que las personerías activas para el año 1976 serían unas 1.100 (20\% menos), la cifra de intervenciones dada por el gobierno militar era prácticamente correcta. Se señalan 28 cuando había 35 sindicatos y federaciones intervenidos entre el 24 de marzo y el 30 de abril 1976, y fueron intervenidos 13 más en el transcurso del mes de mayo. ${ }^{8}$ Téngase en cuenta que el total de intervenciones de 1976 fue de 88, excluyendo la CGT y sin contemplar la designación de "delegados normalizadores" (que elevaría el número a 101 pero fusionaría dos situaciones disímiles). Estos números difieren de los imprecisos "cientos" que se mencionan desde la década del 80 y difieren también de datos de elaboración reciente (Campos, 2008) demostrando que la indagación es aún necesaria para precisar datos que, impresionistas o mal construidos, han alimentado la idea de que el funcionamiento

8. Datos de elaboración propia en base a las Resoluciones del Ministerio de Trabajo, 1976. 
de los sindicatos fue cuasi nulo y que las intervenciones abarcaban al conjunto del movimiento sindical nucleado en la CGT.

El gobierno justificaba estos casos de intervención como respuesta a la corrupción sindical y con el mismo argumento sostenía el encarcelamiento y procesamiento judicial de algunos "prominentes" dirigentes. Declaraba, como cierre, que facilitaría informaciones suplementarias al Comité, algo que efectivamente sucedería.

Puede afirmarse que las respuestas del gobierno estaban orientadas a contener las quejas en Ginebra a sabiendas de que las mismas podian afectar su posición en los foros internaciones. Pero esa tarea no puede desvincularse de la importancia que tenía para la Junta la organización del escenario local, en el que los sindicatos -y la situación de la clase obrera en general- eran considerados elementos de máxima relevancia incluso cuando existían entre las fuerzas criterios disímiles sobre la cuestión.

Ahora bien, ¿cuál fue en ese contexto la actitud de la dirigencia sindical que habia actuado hasta el 24 de marzo en la CGT ahora intervenida y que sabía de la presión que el gobierno estaba recibiendo en la OIT? Partamos de considerar que esa dirigencia no podía menos que intuir que en la estrategia gubernamental de instalación internacional eran un eslabón clave, lo que les aseguraba un espacio de protagonismo.

\section{La contribución patriótica}

Como se señaló, entre marzo y mayo de 1976 fueron en aumento las denuncias en Ginebra ante las que el gobierno presentó una primera respuesta. Además, el Ministerio de Trabajo informó que estaba participando en las reuniones del Consejo de Administración (previas a la Conferencia) por medio de su director de Asuntos Jurídicos. Las dos señales, la respuesta a las quejas y la participación preparatoria, revelaban la intención del gobierno, o al menos de un sector del mismo, de asistir a la Conferencia.

Pero como en las cuestiones de política interna, en el área de política exterior también se presentó tempranamente una ruptura entre "bandos" militares que separaba a quienes rechazaban el diálogo con las organizaciones políticas y sindicales $-\mathrm{y}$, por ende, la participación en la OIT les parecía un contrasentido y hasta un riesgo- y quienes desde la fracción "politicista" veían en la participación la posibilidad de

9. Canelo señala que la fracción politicista era partidaria de un acercamiento con las organizaciones civiles "por considerarlas interlocutoras válidas" para superar el aislamiento del gobierno (Canelo, 2008: 59). Un referente de esta fracción era el propio ministro Liendo, cuyo estilo dialoguista ha sido ya señalado (Novaro y Palermo, 2003; Quiroga, 1994). 
sacar ventajas que fortalecieran al "Proceso". Las diferencias militares al respecto no tardaron en llegar a los diarios de circulación nacional, y un editorial del diario La Nación (17 de mayo de 1976) lo refleja. Alli se señalaba que el Ministro Liendo estaría considerando la agenda de la OIT y se indica que "no estaría decidida aún nuestra participación en razón del desacuerdo entre dos corrientes de opiniones" surgidas en el seno de las Fuerzas Armadas. Según el editorial, un ala sostendría que era mejor no participar "para evitar que la izquierda, especialmente la radicalizada (...) cuestione a nuestra delegación por los hechos institucionales que se sucedieron a partil del 24 de marzo último. Los problemas argentinos -se acotó- los debemos resolver los argentinos, aquí, en el territorio nacional". El mismo editorial indicaba que la otra tendencia "juzga que nada debe temerse ni ocultarse ante cualquier requerimiento que pudiera formularse sobre la realidad imperante en estos dias" y que el gobierno podria "demostrar" la justicia de las medidas tomadas interviniendo en Ginebra. La naturaleza del viaje además comprometía el acuerdo de al menos dos de las Fuerzas (del Ejército, a cargo del Ministerio de Trabajo y de la Marina, que tenia bajo su égida a la Cancillería).

Por si las impugnaciones externas fueran insuficientes, el editorial indica que el cuadro era más complejo aún porque cabía la pregunta sobre a quién habría de confiársele la representación sindical cuando "los mandatos de todos los dirigentes caducaron a raíz de una de las primeras medidas del actual gobierno. Claro, el impedimento es relativo" señala "pues todavía quedan dirigentes con facultades muy limitadas, pero dirigentes al fin". El último apartado del editorial es aún más informativo de los debates y decisiones de aquellos meses. Destaca que el Ministerio de Trabajo, pese a las dificultades que supuestamente enfrentaba el gobierno, había dedicado su esfuerzo a enderezar la estructura sindical y restablecer el orden alterado "desde 1946". Señalaba que para cumplir las metas del gobierno sería necesario introducir innovaciones en las leyes laborales, cambiar "la actitud mental" de los hombres que aspirasen a ocupar lugares sindicales, "sanear" las instituciones y "en resumen, revertir la conformación sindical", algo que requería paciencia y no apresuramiento.

El 20 de mayo, tres días después de aquel editorial, la prensa informó que se había abierto el proceso de elección de delegados obreros para ir a la OIT. Los secretarios generales de los sindicatos se reunirian con el Estado Mayor y el coronel Pita (entonces interventor de la CGT), agrupados en seis grupos ${ }^{10}$ en los que no participarian los representantes de gremios intervenidos.

10. La conformación de estos grupos (también llamados "grupos operativos") fue 
La reunión fue la primera formal y colectiva entre el gobierno militar y los sindicalistas aunque hay que considerar que los encuentros fueron una constante desde marzo (especialmente con militares a cargo de empresas intervenidas, regionales de la CGT o de "zonas de actuación" militar). Así lo atestigua el encuentro entre el interventor de Encotel y los dirigentes del área (R. Baldassini por el FOECYT, A. Cuchetti de AATRA y H. Maffia por el Personal Jerarquizado) el 25 de marzo, o la convocatoria "ante requerimientos sindicales" del comandante de la subzona 51 (Bahía Blanca y alrededores) al día siguiente. ${ }^{11}$ De mayor magnitud pero escasamente documentado es el plenario realizado en la Federación de Trabajadores de la Sanidad donde se constituyó una "comisión de enlace" de 64 sindicatos "autoconvocados para colaborar con la intervención de la CGT" (Senén González y Gallo, 1984: 59; notas personales de Senén González presentes en el Archivo del Sindicalismo Argentino, en la Universidad Torcuato Di Tella, y Clarín, 22 de mayo de 1976).

La reunión convocada por el gobierno constituía una primera oportunidad de expresar públicamente el juicio sindical sobre los sucesos desencadenados el 24 de marzo, y la masiva participación era en sí misma un indicador de la disposición al diálogo que se había manifestado en el plenario informal en Sanidad. Los dirigentes sin embargo decidieron no hacer declaraciones al ingresar al edificio de la intervenida CGT, y fue Liendo quien difundió el objetivo de la reunión: acordar con la dirigencia sindical la participación en la Conferencia. Los sindicalistas no objetaron ni el objetivo general de la reunión ni el mecanismo dispuesto, pero sí presentaron algunas divergencias sobre quiénes debían ser los enviados. De los seis grupos conformados, según informa la prensa, tres designaron su candidato a viajar a Ginebra en ese mismo momento (los grupos dos, tres y cinco) y los otros tres grupos pidieron un cuarto intermedio que les fue concedido y que cumplieron, elevando candidatos después de una segunda reunión (Clarín y La Nación, 27 de mayo de 1976). ${ }^{12}$ Con 18 candidatos indicados y siguiendo el mecanismo dispuesto, Liendo

dispuesta por la intervención de la CGT para esta primera reunión y su existencia perduró en los años siguientes cuando se dispuso reunir a los sindicalistas. Un detalle de los integrantes que integraban cada grupo en 1976 puede verse en el Anexo que acompaña éste artículo.

11. Servicio de Documentación e Información Laboral, editado por Leonardo Dimase, Informe Laboral, abril de 1976.

12. En uno de los grupos no hubo acuerdo en la designación de un representante para enviar a la OIT proponiéndose todos como candidatos pues todos se consideraban "igual de idóneos" (La Nación, 21 de mayo de 1976). Se trata del grupo 6, donde se encontraba la única mujer de la reunión (Cristina Arizaga, de la Unión de Artistas de Variedades). 
seleccionó con Videla la comitiva sindical integrada por un representante titular, un suplente y los asesores de la delegación. Uno (según La Nación) o dos (según Clarin) de los seis grupos habria condicionado la designación de candidatos a un acuerdo con los militares sobre tres puntos: liberación de sindicalistas "que no tengan causa pendiente", ${ }^{13}$ fin de la intervención de la CGT en un plazo de 180 dias y devolución de los sindicatos (La Nación, 22 de mayo de 1976).

Esta posición, señalan ambos diarios, estaría vinculada al grupo "de los 8" asociado al peronismo antiverticalista ${ }^{14}$ y sería opuesta a la del grupo mayoritario autoconvocado en Sanidad, que estaba decidido a avanzar en la colaboración con el gobierno "sin hacer condicionamientos" (Clarín, 22 de mayo de 1976). Era ya público que este último grupo quiso mantener un contacto permanente con la intervención militar de la CGT "y hasta tuvo el propósito de proponer una comisión asesora para que colaborara con el delegado militar en la central obrera" ( $\mathrm{La}$ Nación, 22 de mayo de 1976), algo que los militares rechazaron y que no convencía a algunos referentes de "los 8". La impugnación central giraba en torno a las diferencias entre "los 8" y el sector dominante en la CGT y las 62 organizaciones que, decían, los había excluido y pecaba de "no consultar con los cuerpos orgánicos" antes de actuar (Clarín, 22 de mayo de 1976). Lo que pesaba era la disputa entre los grupos de la dirigencia sindical heredada del período anterior, disputa que se ponía en evidencia en las distintas tácticas de acercamiento-competitivas entre sí- de los sectores gremiales para con los militares. Es necesario señalar que había al menos un miembro de "los 8 " en cada grupo de esa primera reunión, lo que muestra que el cuestionamiento no fue realizado en forma simultánea por todos ellos, aunque el grupo mantuvo su presencia como tal incluso cuando se integró en la denominada "Comisión de los 25" (Senén González y Gallo, 1984: 83-101).

Como se mencionó, el moderado condicionamiento de esos grupos se diluyó en los días siguientes pues todos designaron representantes ante escribano (y entre los propuestos aparecieron miembros vinculados al antiverticalismo), dándole una sólida apariencia democrática a la delegación sindical que sería la llave del éxito para la estrategia gu-

13. Un análisis de todo el período indica que cuando los dirigentes sindicales solicitaban "la libertad de sindicalistas" se referian, exclusivamente, a los dirigentes peronistas detenidos a disposición del Poder Ejecutivo y no a la detención de delegados de fábrica, miembros de comisiones internas y activistas. Hay excepciones muy puntuales a esta generalización.

14. El antiverticalismo surge asociado a Victorio Calabró (Antúnez, 2013). Aquí se lo refiere como agrupamiento dirigido principalmente por Donaires (papeleros). Ver entre otros Senén González (1971), Senén González y Gallo (1984), Zorrilla (1983), Fernández (1985). 
bernamental en Ginebra. Lo cierto es que entre los dirigentes sindicales había diferencias, pero "ambas corrientes coinciden en la necesidad de alinear al movimiento obrero junto a las fuerzas armadas" (Clarin, 20 de mayo de 1976).

En la evaluación de lo sucedido Liendo manifestó que la reunión "revela de por sí una preocupación de los dirigentes sindicales por la participación del sector y del país en esa Conferencia" (La Nación, 22 de mayo de 1976). Lo mismo decía pocos días después el mismo diario señalando que la convocatoria "cumplió sus objetivos" pues no se aceptaron condicionamientos y se evidenciaba que los gremialistas "tienen plena conciencia de que irán a Ginebra conociendo el estado de emergencia que vivimos y la necesidad de explicar que habrán de sumarse al proceso de reorganización del país" (La Nación, 27 de mayo de 1976).

El primer encuentro público entre los dirigentes sindicales y los representantes del gobierno militar lo confirmaba, dejando en evidencia la voluntad de colaboración del sindicalismo ante la situación que enfrentaba el nuevo gobierno en la OIT. No escapaba ni a unos ni a otros el especial valor que adquiría en ese marco la participación sindical, ni lo que implicaba la aceptación de la situación interna con un tácito consenso. Era una lectura lógica de lo sucedido y así lo reflejaba un nuevo editorial de La Nación indicando que "sin necesidad de hilar demasiado fino, debe pensarse que los dirigentes acudieron a la CGT porque están de acuerdo con las leyes del juego, esto es, con las pautas que quedaron trazadas a partir del 24 de marzo pasado. Implícitamente - puede puntualizarse- aceptaron los actos fundamentales del gobierno militar (...) Admitirán también que los problemas que nos aquejan deben dilucidarse y resolverse entre nosotros y no ser llevados a ningún foro internacional para su exposición ante extraños" (La Nación, 24 de mayo de 1976). No se equivocaba el editorialista, el consenso y la participación sindical tenía implicancias externas e internas y constituía un "tesoro" para la Junta al mismo tiempo que le permitía al Ministerio de Trabajo demostrar que su estrategia de diálogo había superado con éxito la primera etapa.

\section{En tierras suizas}

La prensa difundió las declaraciones sindicales realizadas en Ezeiza por R. Baldassini (el seleccionado como titular de la delegación acompañado por R. Valle como suplente y por R. Elorza, H. Barrionuevo, R. Valle, R.J. Pérez, Horvart y Demetrio Lorenzo como asesores), que afirmó que "la delegación argentina tiene ya tomada su posición para este evento internacional y va a hablar con absoluta realidad de lo que pasa en el país. Tenemos fe en que los problemas argentinos los solucionaremos 
dentro de nuestras fronteras y, eventualmente, se va a pedir la solidaridad internacional en el supuesto de que nuestras fuerzas flaqueen o haya hechos manifiestos que hagan imposible o negativa la solución de los problemas nacionales" (La Nación, 1 de junio de 1976). ${ }^{15}$

Las declaraciones fueron más conciliadoras aún cuando Baldassini tocó suelo europeo. Allí indicó que "nuestro sector marginará reservas mentales y actuará con grandeza y auténtica vocación de integración nacional para lograr, como una contribución patriótica, la pacificación del país y el bienestar del pueblo" (La Nación, 14 de junio de 1976). En la evaluación posterior el sector sindical insistió en la idea dejando claro que los problemas argentinos se resolverian "entre argentinos" según informa Clarín (23 de junio de 1976) que habla del éxito y las expectativas del "Operativo Ginebra".

La prensa indicó así las intervenciones de los representantes argentinos pero filtró un equívoco cuya valoración es interesante. Según los reportes (que han replicado con matices y algunos errores entre otros Abós, Fernández y, citando a éstos, muchos como Pozzi o Munck), Baldassini habría pedido por el "restablecimiento de las estructuras sindicales" y Liendo habría difundido las bases de la reorganización en marcha. En realidad estas declaraciones no se realizaron en la Conferencia anual (de la que quedan registros taquigráficos) sino en una conferencia paralela, la Conferencia Tripartita Mundial sobre Empleo, donde estaban excluidos los problemas de libertad y democracia sindical.

La decisión no puede haber sido casual. En la Conferencia anual se debatía el caso chileno y tenían voz las federaciones internacionales que cuestionaban la situación del país. Incluso figuraba acreditado como miembro por la Confederación Mundial de Trabajo Raimundo Ongaro ${ }^{16}$ (ver Actas de la sexagésima primera reunión de la OIT, OIT, 1977), cuyo rol en el armado del caso 842 es indubitable, esto asociado a la acción de denuncia en el exterior de los exiliados sindicales organizados en torno al Centro Sindical por los derechos de los trabajadores en Argen-

15. Las mismas se vieron tenuemente conmovidas por el secuestro de coronel Pita, sucedido el 30 de mayo cuando Liendo subía a su avión como representante del nuevo gobierno. Es presumible que el secuestro, que fue atribuido al Partido Comunista Maoísta Leninista (PCML), se haya realizado ese mismo día asociado a la visibilidad que tenía el viaje a la OIT aunque no hay investigaciones académicas sobre el mismo. En diciembre Pita huyó de su cautiverio y producto de su auto liberación fue ascendido a general (ver legajo personal, Archivo General del Ejército). Ante los hechos un numeroso grupo de dirigentes sindicales se entrevistó con el nuevo interventor de la CGT (Porcile) para expresarle "su satisfacción por la evasión protagonizada por el coronel Pita", afirmando el repudio que profesan los trabajadores argentinos a la subversión (La Nación, 8 de diciembre de 1976).

16. Incorporando como espacio el internacional se evidencia el peso que tuvo la campaña obrera y sindical contra la dictadura en el exterior. 
tina y Latinoamérica (CS), y el Colectivo de Trabajadores y Sindicalistas Argentinos en el Exilio (ver Parcero, Helfgot y Dulce, 1985; Basualdo, 2006; Dawyd, 2014).

La delegación sindical argentina no habló en la Conferencia, no remitió información alguna al Comité de Libertad Sindical ni realizó comunicaciones específicas sobre el tema al Director General o a otros activos miembros del caso, por el contrario se integró silenciosamente al trabajo en comisiones. El plan proyectado por el gobierno y asistido por los dirigentes sindicales resultaba así exitoso, lo que implicaba una señal de gran valor internacional indicando que se "recuperaba" un clima de "normalidad".

Ahora bien, ¿cómo se explica que no hubiera otras quejas en el recinto por la situación argentina, cuando las mismas estaban ya formalizadas ante el Comité de Libertad Sindical y estaban presentes en el recinto al menos algunos de los destacados denunciantes? La respuesta se explica en parte por los mecanismos de la OIT (no había sobre esto un informe del Comité a la Asamblea producto de los tiempos necesarios para su inclusión $)^{17}$ y en parte por la forma en la que se logró la acreditación de la delegación nacional y el peso que efectivamente tenía la "contribución patriótica" del sindicalismo en la coyuntura de instalación del "Proceso" ante los organismos internacionales.

Estando presentes los delegados sindicales "legítimos" y optando ellos por el silencio, la cuestión quedaba circunscripta a los tiempos y trámites del Comité de Libertad Sindical y en ese Comité el gobierno realizaba una estrategia de dilación y repregunta sobre cada persona o violación denunciada, usando todos los mecanismos existentes a su entero favor. Ningún sindicato argentino había expresado ninguna denuncia, preocupación o testimonio ante la Comisión ni antes de la Conferencia, ni durante la misma y sólo lo harian posteriormente sobre casos puntuales algunos sindicatos nacionales, pero no en 1976 (ver A-OIT, Caso 842, 1/1976, 1/1984).

La clave de la respuesta es entonces doble: la acreditación sindical "democrática" y el silencio de la delegación argentina permitieron que el "Proceso" no sufriera las consecuencias de la rápida reacción internacional que despertó. La actitud de la dirigencia sindical constituyó una deliberada muestra de apoyo al gobierno militar, no sólo por no expresar ninguna consideración sobre la situación del país ante la Asamblea anual sino por haberse mantenido al margen -e incluso hostiles- a todo lo relacionado con las denuncias tramitadas en el Caso 842. Su actitud no fue, insistimos, la de un disimulado colaboracionismo sino la de una

17. El primer informe sobre el caso se publicaria en 1977 (ver OIT, Boletín Oficial, vol. LX, 1977, serie B). 
dirigencia politicamente formada (que había participado en forma casi continua en la OIT desde su fundación) que actuaba de acuerdo a sus posiciones e intereses.

En el balance gubernamental de la participación argentina todo fue festejo. Liendo habló de "la calidad de los enviados" y de la inobjetable representatividad sindical (La Opinión, La Nación, Clarín, 16 de junio de 1976) mientras que la prensa reiteraba al unísono la "coherencia" de todos los sectores. No era para menos, el gobierno militar había contenido con su alianza tripartita las críticas existentes por la situación represiva y sorteado con su presencia los cuestionamientos en marcha, algo posible sólo por la colaboración sindical tal y como se dio.

Finalizado el "operativo Ginebra" la contribución tuvo sus frutos. Los sindicatos realizaron una serie de nuevos plenarios en la CGT -siguiendo el esquema de grupos ya dispuesto- donde pudieron evaluar lo actuado y "deliberar" sobre las preocupaciones del momento: libertad de los dirigentes sindicales presos, cese de las intervenciones y esquema de participación en el "Proceso" (La Nación y Clarín, 1 de julio de 1977). El primer gesto de diálogo lo dio Liendo, que a dias de su llegada comenzó una serie de reuniones con figuras destacadas del medio laboral (ex ministros, abogados y, finalmente, sindicalistas) para avanzar en la reforma de la ley sindical que permitiria la elecciones de delegados y comisiones internas en 1976 y de conducciones gremiales en 1977 (Clarin, 2 de julio de 1976).

Le retribución llegó a su punto máximo el 5 de julio, cuando la delegación tripartita se entrevistó en la Casa Rosada con el presidente de facto. En la entrevista con Videla, Baldassini destacó el trabajo intenso "en comisiones técnicas" y en la Conferencia sobre Empleo y expuso "el tema de la normalización sindical" ratificando las "aspiraciones" del sector sobre normalización de sindicatos y obras sociales, libertad de los presos y libre actividad sindical. Incluyó dos temas que no habían sido señalados por los sindicalistas y que "desaparecerian" de la agenda poco después de éste encuentro: los cesantes en la administración pública y la Ley de Contratos de Trabajo. Videla respondió que habría avances sobre las normas necesarias "en el momento oportuno" y les agradeció la misión cumplida. Al salir de la reunión Baldassini destacó que los sindicatos "serán un instrumento necesario para la reorganización nacional" (La Nación y Clarín, 6 de julio de 1976).

Unos días más tarde, el 12 de julio, el Poder Ejecutivo proclamó a pedido de Liendo una ley por la que suspendía la realización de actos eleccionarios y la celebración de asambleas y congresos en los sindicatos (ver Ley 21.356, Boletín Oficial del 27 de julio de 1976). A primera vista la ley podia interpretarse como un brusco giro en la política gubernamental con los sindicatos (y de hecho fue mencionada innumerables veces 
de ese modo), pero una lectura atenta nos permite recuperar un matiz significativo. Con la ley, el Ministerio de Trabajo quedaba autorizado a prorrogar los mandatos de las Comisiones Directivas de los sindicatos y "por otra parte" a reemplazar trabajadores que se desempeñaban hasta entonces como delegados, miembros de Comisiones Internas o de sección en los lugares de trabajo. La "separación" dispuesta para el tratamiento de la dirigencia sindical y de la base obrera movilizada cobraba fuerza de ley.

Hasta aquí hemos atendido las características que tuvo la actividad de la dirigencia nucleada en la CGT, o sea, de aquella identificada con el peronismo que hegemonizó la central desde su normalización en 1970 (Dawyd, 2010). Sin embargo para completar esta revisión es necesario señalar que un grupo de sindicatos "no confederados" desarrolló también en este período una enérgica actividad, especialmente porque vieron la llegada militar como una posibilidad para ocupar espacios que dejaría vacante "la limpieza de los sindicatos". Esta actitud adoptaron los denominados 32 Gremios Democráticos bajo la dirección del radical Juan Carlos Brunetti, de la Asociación de Empleados de Despachantes de Aduanas. En mayo el agrupamiento expresó su disconformidad con la convocatoria a los gremios de la CGT para participar en la OIT, incluso cuando estaba en debate si habría un séptimo grupo para los no confederados -grupo que no se constituyó en ese momento pero que sí lo hizo después, para recibir el informe posterior de la delegación sindical $^{18}$ (entre otras menciones, La Nación, 20 de mayo de 1976; Clarín, 8 de julio de 1976. Ver también la carpeta "1976" del Archivo Senén González ya mencionado).

Cuando producto del entendimiento en Ginebra todo indicaba que habría una nueva ley sindical para una relativamente rápida normalización, Brunetti acercó sus ideas a la Comisión de Asesoramiento Legislativo

18. De aquí el equivoco, común en la literatura, sobre si se reunieron seis o siete grupos en la reunión de mayo. El denominado "grupo 7" se reunió por primera vez el 6 de julio con algunos de los representantes que viajaron a Ginebra. Estaban presentes el Centro de Patrones de Cabotaje; Personal de la Dir. de Ferrocarriles; Centro de Comisarios Navales; el Sindicato de Empleados y Obreros de la Enseñanza Privada; de Feriantes del Gran Buenos Aires; de Jardineros; la Sociedad Argentina de Locutores; el Sindicato de Maestranza; de Obreros y Empleados del Mercado de Liniers y de Empleados de la Industria Cinematográfica. Estaban citados a este grupo pero no concurrieron a la reunión: Empleados de Agencias e Informes; Sindicato de Choferes de Automotores de Alquiler; de Empleados distribuidores de cinematógrafos; de Docentes Particulares; de Obreros y Empleados del Fibrocemento; de Obreros Lustradores de Calzado; de Obreros y Empleados del Mercado Nacional de Avellaneda; de Empleados Joyeros y Relojerías; la Asociación de Personal de Sanidad Ferroviaria; los Conductores Fusionados de la República Argentina y el Sindicato Obreros de la Industria Sanitaria. 
(CAL) donde se trataba el tema. La misma tenía la forma de una declaración de la Junta Ejecutiva Nacional bajo el título de "Consideraciones sobre las modificaciones a la ley de asociaciones profesionales" y estaba fechada el 12 de julio de 1976 (ver documentos de la CAL en el Archivo General de la Nación). El documento exponía que era necesario asegurar el desenvolvimiento democrático de los sindicatos "propendiendo a acostumbrar a los trabajadores a ejercitar y recuperar los hábitos de coexistencia constructiva" inspirados "en las organizaciones obreras ejemplares de Alemania Occidental, Israel y Estados Unidos”. Instaba a la CAL además a que "ningún riesgo circunstancial influyera para que se perpetúe el verticalismo funesto que ha tenido la CGT" pues sostenía que el deber del momento era defenderse de las "acechanzas nazi comunistas [...] y del axioma totalitario".

Este grupo, aunque se reunió posteriormente en la CGT y desarrolló una intensa actividad -empero siempre marginal- mantuvo posiciones muy críticas a Liendo por su diálogo "con los peronistas" y por lo que entendía como concesiones a un sector que debería erradicarse. Constituyen un sector con larga trayectoria en el mundo sindical argentino y poca representatividad, cuyos posicionamientos no pueden fundirse con los de la dirigencia sindical cegetista.

\section{Consideraciones finales}

La aproximación aquí realizada permite, en primer lugar, reafirmar la importancia que tuvo la participación en la OIT durante la instalación internacional del "Proceso" y la trascendencia de la misma como organizadora de las relaciones entre la dirigencia sindical y el gobierno militar en 1976. A lo largo del artículo se demuestra la fertilidad que tiene, aún hoy, reexaminar esa relación reabriendo un campo donde es necesaria una interpretación integradora.

Como se dijo, este trabajo cuestiona la imagen de una dirigencia sindical que enfrentó "como pudo" a un "corsé represivo" que impuso la "quietud sindical" hasta, por lo menos, 1979. Esa lectura, propuesta en principio por Abós pero ampliamente aceptada, exime a la dirigencia sindical asignándole a lo sumo la responsabilidad de haber actuado bajo el paradigma de un vago colaboracionismo propio de los diálogos corporativos de la década del 60.

Por el contrario, la participación en la OIT puede utilizarse como muestra y sintesis adecuada de la lectura que hizo la dirigencia sindical y de los objetivos prioritarios que articuló más o menos abiertamente en el primer año del gobierno militar. Esto a sabiendas de que la actitud de apoyo e integración en el proceso político no fue suficiente para encauzar "la cuestión sindical" y que las últimas semanas de diciembre verian 
la ruptura de la concordia lograda. Se habian interpuesto en el entendimiento al menos tres situaciones que aquí no fueron contempladas pero cuya importancia no subestimamos: la persistencia del conflicto en la base obrera -especialmente de los sectores automotrices, donde habia un fuerte retroceso en cantidad de puestos de trabajo (Schneider, 2000)-; las resistencias en el área del Estado a la aplicación de la Ley 21.476, por la que anulaban derechos obtenidos en negociaciones previas a 1976 -especialmente el impulso y despliegue del conflicto entre el gobierno y los trabajadores de las empresas de electricidad y su sindicato (Ghigliani, 2011)-, y el recrudecimiento de la interna militar que enfrentaba la estrategia de Liendo contra la de un sector "duro" que sostenía la necesidad de cortar vínculos con la dirigencia sindical peronista e impedir la actividad sindical siguiendo una aplicación rígida de las normas dispuestas hasta entonces (Novaro y Palermo, 2003; Canelo, 2008).

Pero nuestra evaluación no puede restringirse a los vaivenes posteriores que tuvo esa concordia y mucho menos a una "redención" de esa dirigencia por decisiones ulteriores. Por el contrario, hay que afirmar que la dirigencia sindical tuvo una aguda lectura política de la situación nacional e internacional que enfrentaba el gobierno en 1976 y actúo (lejos de toda quietud) articulando una moderada defensa de los sindicatos y en busca de una participación política nacional avalando la denominada "lucha contra la subversión". Esto sin dejar de reconocer que ya en 1976 existieron disimiles tácticas de acercamiento -insistimos, competitivas entre sí- de los sectores gremiales para con los militares.

\section{Anexo. Listado de sindicatos de los cinco grupos}

\section{GRUPO I}

Sindicatos presentes: - Asoc. de Supervisores de la Industria Metalúrgica (Guillermo) • Asoc. Mutual y Gremial de Empleados de Escribanías (Carballo) • Fed. de Obreros y Empleados de la Industria Aceitera (Gaetini) • Fed. de Obreros y Empleados de la Industria del Papel (Donaires)* • Fed. de Peluqueros y Peinadores (Dorato) • Fed. de Trabajadores de Edificios de Renta y Horizontal (Fernández Alonso) $\bullet$ Fed. Obrera Cervecera (Barilache)** ${ }^{*}$ Sindicato de Trabajadores Perfumistas (Ferrero) • Sindicato Único de Trabajadores del Espectáculo Público (Barin) • Sindicatos de Obreros y Empleados de Chacinados y Afines (Sotelo) • Unión de Trabajadores de Entidades Deportivas y Civiles (Bejar) ${ }^{* *}$ - Sindicato de Camioneros (Perez) ${ }^{* *}$ - Unión de Trabajadores del Calzado (Marchese)**

Sindicatos ausentes: - Asoc. de Agentes de Propaganda Médica • Fed. de Agentes Viales Unión de Trabajadores Sanitarios • Unión Personal de Casas Particulares

Sindicatos intervenidos: • Asoc. Bancaria • Unión Obrera Metalúrgica 


\section{GRUPO II}

Sindicatos presentes: - Asoc. de Trabajadores de la Industria Lechera (Heiler) • Asoc. Obrera Minera (Ruiz) • Fed. de Trabajadores de Aguas Gaseosas (Rachini)* • Fed. de Trabajadores de Aguas Sanitarias de la Nación (Serpa)** • Fed. de Trabajadores Químicos (Valle)** $\bullet$ La Fraternidad (Cal) • Sindicato de Obreros y Empleados de la Educación y la Minoridad (Contreras) • Sindicato de Obreros y Empleados del Fósforo (Maldonado) - Sindicato Unico de la Publicidad (Cabrera) - Sindicato Unificado Barracas de Lana (Taglia)** • Unión de Trabajadores de Carga y Descarga (Cladera)

Sindicatos ausentes: - Fed. de Trabajadores de la Sanidad • Sindicato de Trabajadores del Automóvil Club Argentino

Sindicatos intervenidos: - Asoc. Obrera Textil • Unión Ferroviaria • Fed. Obrera Ceramista • Fed. Gremial del Personal de la Carne

\section{GRUPO III}

Sindicatos presentes: • Confederación de Empleados de Comercio (Carranza) - Confederación de Obreros y Empleados Municipales (Izzetta)* • Fed. Argentina de Trabajadores del Cuero (Negrete) • Fed. de Trabajadores Jaboneros (Nieto) - Fed. Obreros de la Industria del Vestido (Micó) ${ }^{* *} \bullet$ Fed. Obreros del Tabaco $($ Loiacono)** $\bullet$ Fed. Sindical de Petroleros Privados (Martillotta) $\bullet$ Fed. Única de Viajantes (Diz Rey)** • Sindicato de Empleados del Tabaco (Dighon) • Sindicato del Seguro (Valle)** - Sindicato Obreros de Cementerios (Petrecca) • Unión Obrera Ladrillera (Chain) • Unión Personal de Fábricas de Pinturas (Zambelletti)

Sindicatos ausentes: - Sindicato de Trabajadores del Hielo - Sindicato de Empleados y Obreros de la Cinematografia $\bullet$ Unión Obrera Refractarios

Sindicatos intervenidos: • Sindicato Unidos Petroleros del Estado • Unión de Obreros y Empleados Municipales

\section{GRUPO IV}

Sindicatos presentes: - Asoc. de Capataces Estibadores Portuarios (Ávila) - Asoc. de Personal de Empresas de Seguridad e Investigadores Privados (Méndez) • Asoc. de Viajantes de Comercio (Estévez) • Círculo de Electricistas Navales (Venturini) • Fed. de Trabajadores Fideeros (Barrionuevo)** • Sindicato de Apuntadores Marítimos (Thompson) - Sindicato de Colchoneros (Nieto Maceiras) - Sindicato de Conductores de Taxi (García) - Sindicato de Obreros de Estaciones de Servicios y Garages (Martín) - Sindicato Obreros Marítimos Unidos (Massagatti)** Unión Obrera Molinera (Soberano) • Unión Trabajadores Gastronómicos (Elorza)* - ** • Unión Tranviarios (Ríos)

Sindicatos ausentes: - Fed. Argentina de Empleados de Casinos • Fed. Personal de Vialidad Nacional • Sindicato de Guincheros de Puertos

Sindicatos intervenidos: • Fed. de Sindicatos Unidos Portuarios Argentinos

- Sindicato de Obreros Navales • Unión Obrera de la Construcción 


\section{GRUPO V}

Sindicatos presentes: - Asoc. de Empleados de la Industria del Vidrio (Millán) • Asoc. de Empleados de la Protección a la Aeronavegación (Domínguez) • Asoc. de Trabajadores del Estado (Horvart)** $\bullet$ Fed. Argentina de Personal de la Aeronáutica (Beotegui) • Fed. Argentina de Trabajadores Rurales (Corredera) • Fed. Obreros y Empleados Vitivinícolas (Petrich) • Futbolistas Argentinos Agremiados (Tasdivo) - Sindicato de Obreros de la Industria del Vidrio (Castillo)*-** - Sindicato Único del Neumático (Serrano)** • Unión de Obreros y Empleados Plásticos (Brum) • Unión Sindicatos Industria Maderera (Di Santos)

Sindicatos ausentes: - Asoc. Argentina de Aeronavegantes - Sindicato de Jaboneros • Unión de Recibidores de Granos***

Sindicatos intervenidos: - Fed. Argentina de Trabajadores de Prensa • Sindicato de Mecánicos del Transporte Automotor • Unión de Personal Civil de la Nación • Unión Docentes Argentinos

\section{GRUPO VI}

Sindicatos presentes: • Asoc. Argentina de Actores (Rivera López)** • Asoc. Argentina de Telegrafistas, Radiografistas y Afines (Prado) ${ }^{* *} \bullet$ Fed. Argentina de Personal de Panaderías (López)** ${ }^{*}$ Fed. Argentina de Trabajadores de Imprenta (Marano $^{* *} \bullet$ Fed. de Obreros y Empleados de Correos y Telecomunicaciones (Baldassini)** • Fed. de Obreros y Empleados Mosaístas (Cardozo)** • Fed. de Trabajadores de la Alimentación (Lorenzo)** ${ }^{*}$ Fed. de Trabajadores del Turf (Cocimano) $^{* *} \bullet$ Fed. del Personal Jerárquico de Encotel (Maffia)** ${ }^{*}$ Fed. Obreros del Caucho (Borda)** • Frigorifico Nacional Lisandro de la Torre (Gómez) ${ }^{* *} \bullet$ Sindicato Argentino de Televisión (Ferredás Campos)** • Sindicato de Empleados Textiles (Amono)** $\bullet$ Unión de Artistas de Variedades (Arizaga)** ${ }^{*}$ Unión Obreros Tintoreros (Blanco)**

Sindicatos intervenidos: • Fed. de Obreros y Empleados Telefónicos • Fed. de Trabajadores de Luz y Fuerza • Fed. del Personal de Gas del Estado • Fed. Tucumana de la Industria del Azúcar

* Miembros del agrupamiento "Grupo de los 8".

** Propuestos para participar de la OIT.

*** La Unión de Recibidores de Granos y Anexos de la Republica Argentina, que se consignaba en la prensa como "ausente", se encontraba en realidad en proceso de "normalización" por Resolución n ${ }^{\circ} 87$ del Ministerio de Trabajo del 20 de abril de 1976. Fuente: Elaboración propia.

\section{Bibliografia}

Abós, Alvaro (1984), Las organizaciones sindicales y el poder militar, Buenos Aires: CEAL.

Alexander, Robert J. (2009), International Labor Organizations and Organized Labor in Latin America and the Caribbean: A History, Santa Barbara: ABC-CLIO. 
Antúnez, Damián (2013), "El gobierno bonaerense de Victorio Calabró: entre la intervención federal y el golpe de Estado", PolHis: Boletín Bibliográfico Electrónico, 6 (12): 174-193.

Basualdo, Victoria (2006), "La participación de los trabajadores y sindicalistas en la campaña internacional contra la última dictadura argentina", Sociedad, 25: 197-222.

- (2010), “The ILO and the Argentine Dictatorship (1976-1983)", en Jasmien van Daele (ed.), ILO Histories Essays on the International Labour Organization and Its Impact on the World during the Twentieth Century, Berna-Nueva York: Peter Lang.

Belloni Ravest, Hugo (1969), El sindicalismo argentino en la Organización Internacional del Trabajo, Buenos Aires: s/d.

Brennan, James P., y Mónica Gordillo (2008), Córdoba rebelde: el cordobazo, el clasismo y la movilización social, La Plata: De la Campana.

Campos, Luis (2008), "Estado y sindicatos: un análisis de sus relaciones a partir de los mecanismos de regulación y la conformación de la estructura sindical en Argentina (1943-1988)", tesis de Maestría, FLACSO Argentina.

Canelo, Paula (2008), El Proceso en su laberinto: la interna militar de Videla a Bignone, Buenos Aires: Prometeo.

Dawyd, Dario (2010), "Sindicatos y politica en Argentina, 1968-1970. De la división a la fractura del peronismo", Universidad Nacional de Tres de Febrero, Red de Estudios sobre el Peronismo (en línea).

- (2014), "Raimundo Ongaro, dirigente sindical. Trayectoria de un peronista combativo en contextos de hostilidad (Argentina, 1966-1983)", Abordajes. Revista de Trabajo Social, 2 (1).

Delich, Francisco (1982), "Después del diluvio, la clase obrera", en Alain Rouquié, Argentina, hoy, México: Siglo Veintiuno.

Fernández, Arturo (1985), Las prácticas sociales del sindicalismo: 19761982, Buenos Aires: CEAL.

Ghigliani, Pablo (2011), "El conflicto de Luz y Fuerza de 1976-1977: ensayo de interpretación”, en IV Seminario Internacional Políticas de la Memoria.

Godio, Julio (1980), Historia del movimiento obrero latinoamericano, CaracasMéxico: Nueva Sociedad-Nueva Imagen.

- y Achim Wachendorfer (1986), El sindicalismo internacional en America Latina, Buenos Aires: Fundación Friedrich Ebert.

Guest, Iain (1990), Behind the Disappearances: Argentina's Dirty War against Human Rights and the United Nations, Philadelphia: University of Pennsylvania Press.

Novaro, Marcos, y Vicente Palermo (2003), La dictadura militar, 1976-1983: del golpe de estado a la restauración democrática, Buenos Aires: Paidós.

Parcero, Daniel (1987), La CGT y el sindicalismo latinoamericano: historia crítica de sus relaciones desde el ATLAS a la CIOSL, Buenos Aires: Fraterna.

-, Marcelo Helfgot y Diego Dulce (1985), La Argentina exiliada, Buenos Aires: CEAL. 
Pozzi, Pablo, y Alejandro Schneider (2000), Los setentistas: izquierda y clase obrera, 1969-1976, Buenos Aires: Eudeba.

Quiroga, Hugo (1994), El tiempo del "proceso": conflictos y coincidencias entre politicos y militares, 1976-1983, Rosario: Fundación Ross.

Schneider, Alejandro (2000), "Ladran Sancho...". Dictadura y clase obrera en la zona norte del Gran Buenos Aires", en Hernán Camarero y Pablo Pozzi (eds.), De la Revolución Libertadora al menemismo: historia social y politica argentina, Buenos Aires: Imago Mundi.

Senén González, Santiago (1971), El sindicalismo después de Perón, Buenos Aires: Galerna.

- y Ricardo Gallo (1984), Diez años de sindicalismo argentino, de Perón al proceso, Buenos Aires: Corregidor.

Zorrilla, Rubén (1983), El liderazgo sindical argentino: desde sus orígenes hasta 1975, Buenos Aires: Siglo Veinte.

Zorzoli, Luciana (2015), "La normativa sindical entre la dictadura y el alfonsinismo, propuesta de sistematización", en Alejandro Schneider y Pablo Ghigliani (eds.), Clase obrera, sindicatos y Estado. Argentina (1955-2010), Buenos Aires: Imago Mundi.

$$
* * *
$$

Título: "Operativo Ginebra". The union leadership facing the international system of military dictatorship

Resumen: El artículo analiza los posicionamientos de la dirigencia sindical en el contexto de instalación internacional del gobierno militar considerando especialmente la participación argentina en la Organización Internacional del Trabajo (OIT). En esta revisión se refutarán empíricamente los análisis que sostuvieron que la actividad sindical se vio suspendida por la magnitud de la represión y de las intervenciones contra las organizaciones obreras. Por el contrario se pondrá en evidencia la intensa actividad desplegada por la dirigencia sindical peronista en 1976, y se analizarán sus posicionamientos y expectativas en torno al gobierno de facto.

Palabras clave: Sindicatos - dictadura militar - Organización Internacional del Trabajo (OIT)

Abstract: This paper analyse the different political strategies adopted by the trade union leadership in the context of the international installation of the 1976 militarygovernment, with special attention to what has happened at the ILO Annual Conference. Empiricalevidence against the idea that trade unions activity was "suspended" as a consequence of repression and interventions of working class organisations will be shown. On the contrary the paper shows that there was intense activity by peronist trade union leadership in 1976 and it will analyse its expectations and public statements regarding the military government. Keywords: Unions - Dictatorship - International Labour Organization (ILO)

Recepción: 10 de octubre de 2015. Aprobación: 20 de febrero de 2016. 